\title{
Oncogene-induced DNA damage: cyclic AMP steps into the ring
}

\author{
James A. Fagin ${ }^{1,2}$ and John H. Petrini ${ }^{3}$ \\ ${ }^{1}$ Human Oncology and Pathogenesis Program, ${ }^{2}$ Department of Medicine, and ${ }^{3}$ Molecular Biology Program, Memorial Sloan Kettering Cancer Center (MSKCC), New York, New York, USA.
}

\begin{abstract}
Growth hormone-secreting (GH-secreting) pituitary tumors are driven by oncogenes that induce CAMP signaling. In this issue of the JCI, Ben-Shlomo et al. performed a whole-exome study of pituitary adenomas. $\mathrm{CH}$-secreting tumors had a high frequency of whole chromosome or chromosome arm copy number alterations and were associated with an increase in the tumor protein p53 and the cyclin-dependent kinase inhibitor p21 ${ }^{\text {WAF1/CIP1 }}$, which are findings consistent with induction of a response to DNA damage. Further, treatment of mouse pituitary cells with cAMP pathway agonists in vitro and in vivo elicited biomarkers of DNA replication stress or double-strand breaks. The findings of Ben Shlomo et al. indicate that oncoproteins that drive constitutively high cAMP signaling pathway output in susceptible cell types can elicit DNA replication stress and may promote genomic instability.
\end{abstract}

Pituitary adenoma growth and growth hormone secretion

Pituitary adenomas have a prevalence of approximately 1 in 1000 persons and constitute approximately $15 \%$ of all brain tumors. They are often identified incidentally, are almost invariably benign, and are confined to the sella turcica (1). More aggressive tumors can invade surrounding structures and frequently recur after surgical resection. The accepted definition of pituitary malignancy, however, is restricted to tumors that metastasize craniospinally or to distant sites, and these are exceedingly rare (<0.1/100,000 population) (2). Growth hormone-secreting (GH-secreting) pituitary (somatotroph) adenomas, which give rise to acromegaly or gigantism, represent up to $15 \%$ of all pituitary neoplasms.

There is compelling evidence that constitutive activation of cAMP signaling plays an important role in driving somatotroph adenoma growth and GH secretion.
Salient examples are reflected in patients who develop acromegaly as a result of neuroendocrine tumors that ectopically secrete GH-releasing hormone (GHRH) (3) or because of germline or somatic mutations of canonical signaling effectors in the cAMP pathway. Specific mutations include those of GRP101, an orphan G protein-coupled receptor (4), GNAS, the gene encoding the Gs $\alpha$ subunit of the heterotrimeric $G$ protein that activates the enzyme adenylyl cyclase, which catalyzes the conversion of ATP to $\operatorname{cAMP}(5,6), P K R A R 1 A$, a regulatory subunit of protein kinase $A(7)$, as well as inactivating mutations of $A I P$, the aryl hydrocarbon receptor-interacting protein (8).

\section{A signature of DNA damage response}

In this issue of the JCI, Ben-Shlomo et al. describe the results of whole-exome sequencing of 159 pituitary adenomas, the largest series reported so far. The

Related Article: p. 5738

Conflict of interest: JAF is a consultant for Loxo Oncology, received grant support from Eisai Pharmaceuticals, and is a coinventor of intellectual property focused on HRAS as a biomarker for cancer using tipifarnib, which has been licensed by MSKCC to Kura Oncology. JHP is a consultant for Novus Biologicals and Atropos Therapeutics.

Copyright: @ 2020, American Society for Clinical Investigation.

Reference information: / Clin Invest. 2020;130(11):5668-5670. https://doi.org/10.1172/JCI142237.

study revealed infrequent recurrent single nucleotide mutations or small insertions/deletions, with the exception of $29 \%$ of somatotroph adenomas that harbored activating mutations of GNAS (9). Consistent with prior studies, somatic copy number alterations (SCNAs) were the predominant lesions $(10,11)$ and primarily involved gains or losses of whole chromosomes or chromosome arms, which were particularly enriched in GH- and prolactin-secreting adenomas. The size of the study cohort allowed the researchers to utilize the Kyoto Encyclopedia of Genes and Genomes (KEGG) to analyze single gene pathways lying within these large SCNAs. The KEGG pathway analysis revealed that genes relating to the inherited disease Fanconi anemia were more frequently deleted in secretory adenomas, particularly somatotroph tumors. The genes represented in the KEGG Fanconi anemia pathway, which include BRCA2, BRCA1, REV3L, HES1, and $R M I 1$, would be more accurately defined as components of a signature of response to DNA damage arising during DNA replication. Whereas the Fanconi anemia pathway mitigates the toxicity of DNA regions whose cross strands are covalently linked (interstrand DNA crosslinks), the five genes identified contribute to repairing DNA double-strand breaks (DSBs) (12). Only somatotroph adenomas had increased levels of p53 and $\mathrm{p} 21^{\mathrm{CIP} 1 / \mathrm{WAF} 1}$, consistent with the notion that these cells may manifest more general forms of DNA damage.

When Ben-Shlomo and colleagues treated mouse primary pituitary cultures with forskolin or GHRH to increase cAMP levels, phosphorylated histone protein $\mathrm{H} 2 \mathrm{AX}(\gamma \mathrm{H} 2 \mathrm{AX})$ levels increased. Phosphorylated $\gamma \mathrm{H} 2 \mathrm{AX}$ forms when double-stranded DNA DSBs appear. Longterm treatment of mice with the long-acting GHRH analog CJC-1295 increased $\mathrm{GH}$ levels and pituitary weight, which was associated with an increase in $\gamma \mathrm{H} 2 \mathrm{AX}$ 


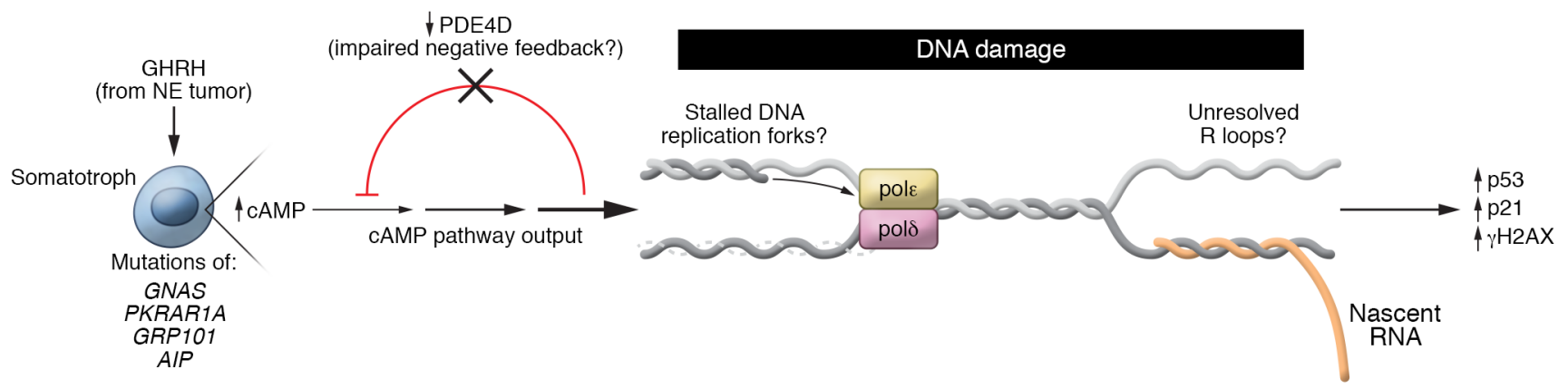

Figure 1. Potential mechanisms for induction of the DNA damage response pathway in GH-secreting pituitary adenomas. Somatotroph tumors harbor widespread chromosomal copy number abnormalities. The major disease drivers include germline or somatic mutations of signaling effectors in the cAMP signaling pathway or, less commonly, ectopic secretion of GHRH by neuroendocrine (NE) tumors. The mutant proteins activate cAMP signaling, which may be further augmented through impaired negative feedback by downregulation of the phosphodiesterase PDE4D. Ben-Shlomo et al. (9) showed that human $\mathrm{CH}$-secreting tumors had an increase in $\mathrm{p} 53$ and the cyclin-dependent kinase inhibitor p21 ${ }^{\mathrm{CIP} / \text { WAF1 }}$, consistent with a response to DNA damage. These results were supported by in vitro and in vivo experiments that showed that potent activation of cAMP signaling increased $\gamma \mathrm{H} 2 \mathrm{AX}$ and Olive tail moment in comet assays in mouse pituitary cells. The mechanisms that induce the DNA damage response are unknown but could involve DNA replication stress and/or collisions between transcriptional and DNA replication complexes, such as stalled DNA replication forks or unresolved R loops. DNA damage occurs in response to illegitimate activation of a variety of oncoproteins. The finding that cAMP signaling is implicated in the induction of the DNA damage response may be relevant to other endocrine cell lineages in which the CAMP pathway regulates cell proliferation and hormone gene expression.

and Olive tail moment in comet assays, a measure of noncontinuous DNA strands. Although these data could indicate an induction of DNA DSBs or DNA replication stress, $\gamma \mathrm{H} 2 \mathrm{AX}$ can also be present during other biological processes, such as normal DNA replication, gametogenesis, or histone deacetylase inhibition $(13,14)$. Similarly, a comet assay is a useful reporter of DNA damage, but the assay is also affected by the fraction of cells in $S$ phase. Both damaged DNA and DNA replication intermediates, such as unresolved Okazaki fragments, are noncontiguous DNA species, and both contribute to the Olive tail moment (15). Hence, the increased $\gamma \mathrm{H} 2 \mathrm{AX}$ levels and Olive tail moments observed by Ben-Shlomo et al. may reflect both DNA damage and increased levels of S-phase cells. Although each of these experiments in isolation is subject to alternative interpretations, taken together with the evidence that $\mathrm{GH}$ adenomas have increased p53 and p21 $1^{\mathrm{CIP} 1 / \mathrm{WAF} 1}$ levels, these data make a convincing case that tumoral somatotrophs are engaged in a response to DNA damage (Figure 1).

The unresolved question is, what might be triggering this DNA damage response process? Oncogenes that drive tumor cell growth co-opt the activity of key signaling effectors that regulate proliferation. RAS and BRAF, which signal primarily through the canonical MAPK pathway, regulate the growth of many cell lineages, and their oncogenic mutants disrupt homeostatic negative feedback inputs that dampen and ultimately extinguish pathway output. This unconstrained signaling results in DNA replication stress, which may include replication fork collapse and the generation of double-strand DNA DSBs. This DNA damage, in turn, leads to increased levels of $\gamma \mathrm{H} 2 \mathrm{AX}$ and may ultimately trigger senescence (16-19).

\section{Conclusions and considerations}

As Ben-Shlomo and colleagues point out, the lack of appropriate model systems detracts from the ability to explore the mechanisms accounting for DNA damage in somatotroph tumor cells in greater detail (9). As in other endocrine cell types, cAMP was the key second messenger driving growth and hormone production in $\mathrm{GH}$-secreting cells. Upon stimulation with forskolin or CJC-1295, there was a chronic and sustained increase in cAMP levels (9). The cyclic nucleotide phosphodiesterases (PDEs) are a large family of enzymes that break down cAMP and cGMP (20). Although some of the PDEs have been found to be overexpressed in pituitary tumors (21), Ben Shlomo et al. found that expression of PDE4D, which specifically degrades cAMP, was decreased in human $\mathrm{GH}$-secreting adenomas (9). Conversely, autonomously functioning thyroid adenoma (AFTA), an analogous benign tumor type also driven by oncogenes that constitutively increase
cAMP levels $(22,23)$, shows up to ninefold higher PDE4D levels when compared with levels in normal thyroid tissue (24). This increase in PDE4 levels points to the presence of a functional negative feedback pathway in AFTAs that is disabled in GH-secreting pituitary tumors, raising the possibility that unconstrained cAMP-driven signaling may trigger DNA hyperreplication in somatotrophs. This hyperreplication would be consistent with the indices of DNA damage response activation observed (9).

Alternatively, unresolved DNA-RNA hybrids ( $\mathrm{R}$ loops) may promote the DNA damage response in tumoral somatotrophs. $\mathrm{R}$ loops form during transcription when nascent RNA hybridizes to its template DNA strand, displacing the complementary single-stranded DNA. When DNA replication forks encounter the transcriptional machinery or the associated $\mathrm{R}$ loop structures, the resulting collisions can cause DNA damage $(25,26)$. In the setting of constitutive cAMP signaling in somatotrophs, in which there is induction of both DNA synthesis and gene transcription, $\mathrm{R}$ loop structures provide a plausible mechanism for inducing the DNA damage response pathway.

Genomic instability is one of the enabling hallmarks of cancer (27). As shown by Ben-Shlomo et al. (9) and others, hormone-secreting pituitary adenomas are characterized by widespread chromosomal abnormalities, yet malignant trans- 
formation is infrequent, as are mutations of TP53 or other tumor suppressors that could allow cells to evade senescence or apoptosis $(9,11)$. Less clear is the possible role of the heterozygous deletions of the DNA repair genes described in this work (9). Presumably the mutations driving constitutive cAMP signaling are early events in pituitary tumorigenesis, with the SCNAs that cause these gene deletions arising later in the course of clonal evolution. One can only speculate as to whether the compound allelic losses of these genes may somehow perpetuate a dysfunctional response to DNA damage that preferentially directs cells toward senescence rather than apoptotic cell death.

\section{Acknowledgments}

JAF and JHP are supported in part by grants from the NIH (CA50706-29 and CA-72597-21, to JAF; GM136278, GM59413, and CA087497, to JHP) and by a National Cancer Institute (NCI) Cancer Center Support Grant (P30 CA08748, to JAF and JHP).

Address correspondence to: James A. Fagin, Memorial Sloan Kettering Cancer Center, 1275 York Ave, New York, New York 10065, USA. Phone: 646.888.2136; Email: faginj@mskcc.org.

1. Lloyd RV, Osamura, RY, Kloppel G, Rosai J. WHO Classification of Tumours of Endocrine Organs, 4th ed. Lyon IARC; 2017.

2. van der Zwan JM, et al. Carcinoma of endocrine organs: results of the RARECARE project. Eur J Cancer. 2012;48(13):1923-1931.
3. Thorner MO, et al. Somatotroph hyperplasia. Successful treatment of acromegaly by removal of a pancreatic islet tumor secreting a growth hormone-releasing factor. J Clin Invest. 1982;70(5):965-977.

4. Trivellin G, et al. Gigantism and acromegaly due to Xq26 microduplications and GPR101 mutation. N Engl J Med. 2014;371(25):2363-2374.

5. Landis CA, Masters SB, Spada A, Pace AM, Bourne HR, Vallar L. GTPase inhibiting mutations activate the alpha chain of Gs and stimulate adenylyl cyclase in human pituitary tumours. Nature. 1989;340(6236):692-696.

6. Weinstein LS, Shenker A, Gejman PV, Merino MJ, Friedman E, Spiegel AM. Activating mutations of the stimulatory $\mathrm{G}$ protein in the McCune-Albright syndrome. N Engl J Med. 1991;325(24):1688-1695.

7. Kirschner LS, et al. Mutations of the gene encoding the protein kinase A type I-alpha regulatory subunit in patients with the Carney complex. Nat Genet. 2000;26(1):89-92.

8. Tuominen I, et al. AIP inactivation leads to pituitary tumorigenesis through defective Gai-cAMP signaling. Oncogene. 2015;34(9):1174-1184

9. Ben-Shlomo A, et al. DNA damage and growth hormone hypersecretion in pituitary somatotroph adenomas. JClin Invest. 2020;130(11):5738-5755.

10. Song ZJ, et al. The genome-wide mutational landscape of pituitary adenomas. Cell Res. 2016;26(11):1255-1259.

11. Bi WL, et al. Landscape of genomic alterations in pituitary adenomas. Clin Cancer Res. 2017;23(7):1841-1851.

12. Ciccia A, Elledge SJ. The DNA damage response: making it safe to play with knives. Mol Cell. 2010;40(2):179-204

13. Cleaver JE, Feeney L, Revet I. Phosphorylated H2Ax is not an unambiguous marker for DNA double-strand breaks. Cell Cycle. 2011;10(19):3223-3224

14. Mirzoeva OK, Petrini JH. DNA replicationdependent nuclear dynamics of the Mre11 complex. Mol Cancer Res. 2003;1(3):207-218.
15. Olive PL, Banáth JP. Induction and rejoining of radiation-induced DNA single-strand breaks: "tail moment" as a function of position in the cell cycle. Mutat Res. 1993;294(3):275-283.

16. Bartkova J, et al. DNA damage response as a candidate anti-cancer barrier in early human tumorigenesis. Nature. 2005;434(7035):864-870.

17. Bartkova J, et al. Oncogene-induced senescence is part of the tumorigenesis barrier imposed by DNA damage checkpoints. Nature. 2006;444(7119):633-637.

18. Di Micco R, et al. Oncogene-induced senescence is a DNA damage response triggered by DNA hyper-replication. Nature. 2006;444(7119):638-642.

19. Fagan-Solis KD, et al. A P53-independent DNA damage response suppresses Oncogenic Proliferation and Genome Instability. Cell Rep. 2020;30(5):1385-1399.e7.

20. Hsien Lai S, Zervoudakis G, Chou J, Gurney ME, Quesnelle KM. PDE4 subtypes in cancer. Oncogene. 2020;39(19):3791-3802.

21. Bizzi MF, Bolger GB, Korbonits M, RibeiroOliveira A. Phosphodiesterases and cAMP pathway in pituitary diseases. Front Endocrinol (Lausanne). 2019;10:141.

22. Parma J, et al. Somatic mutations in the thyrotropin receptor gene cause hyperfunctioning thyroid adenomas. Nature 1993;365(6447):649-651.

23. Lyons J, et al. Two $G$ protein oncogenes in human endocrine tumors. Science. 1990;249(4969):655-659.

24. Persani L, et al. Induction of specific phosphodiesterase isoforms by constitutive activation of the cAMP pathway in autonomous thyroid adenomas. J Clin Endocrinol Metab. 2000;85(8):2872-2878.

25. Crossley MP, Bocek M, Cimprich KA. R-loops as cellular regulators and genomic threats. Mol Cell. 2019;73(3):398-411.

26. García-Muse T, Aguilera A. R Loops: from physiological to pathological roles. Cell. 2019;179(3):604-618.

27. Hanahan D, Weinberg RA. The hallmarks of cancer. Cell. 2000;100(1):57-70. 